\title{
Response of Wheat Plants to Application of Selenium and Humic Acid under Salt Stress Conditions
}

\author{
Mona, I. Nossier, Sh. M. Gawish, T. A. Taha and Manal Mubarak \\ Soils Department, Faculty of Agriculture, Ain Shams University, Cairo, Egypt
}

\begin{abstract}
GELENIUM is an essential element for humans, animals and some species of microOorganisms; however, in higher plants the role of selenium is still unclear. The present investigation was carried out to study the response of wheat plants to selenium application in presence of humic acid under salt conditions, the study included evaluation for the impact of this response on productivity of wheat plants. To achieve this goal two germination experiments were performed in order to choose and define the best concentrations of selenium and humic aid.

Wheat grains were germinated on different concentrations from sodium selenite $(5 \mu \mathrm{m} / \mathrm{l}$ $10 \mu \mathrm{m} / \mathrm{l}-20 \mu \mathrm{m} / 1-40 \mu \mathrm{m} / 1-80 \mu \mathrm{m} / \mathrm{l})$ and humic acid $(0.5 \mathrm{gm} / 1000 \mathrm{ml}-1 \mathrm{gm} / 1000 \mathrm{ml}-2 \mathrm{gm}$ $/ 1000 \mathrm{ml}-1 \mathrm{gm} / 100 \mathrm{ml}-5 \mathrm{gm} / 100 \mathrm{ml}-10 \mathrm{gm} / 100 \mathrm{ml}-20 \mathrm{gm} / 100 \mathrm{ml}-40 \mathrm{gm} / 100 \mathrm{ml}$ ) after soaking the grains for 6 hours to select the most suitable concentrations. Wheat grains were then grown on sandy soil, under different concentrations from mixtures consisting of the best suitable concentrations of both selenium and humic acid under Salt Stress conditions. Based on the results of above preliminary experiments, field experiments were using performed saline soil.

Results showed that soaked wheat grains in different concentrations of mixtures of selenium and humic acid led to increases the percentage of germination as well as length both shoot and root along with their dry matter contents, were also favored total soluble sugars.

Results also revealed that used mixtures of selenium and humic acid in saline soil led to increases resistance of plants to salinity conditions, therefore decreasing the content of proline compared to control, in case of wheat grains, the treatments also improved the proportion of protein and selenium compared to the control.
\end{abstract}

Keywords : Wheat, Sodium selenite, Salinity, Humic acid.

\section{Introduction}

Selenium is one of the "essential" nutrients for human, meaning that our bodies cannot make it, and so we have to get it from our diet. Without it the heart, joints, eyes, immune system or reproductive system may suffer. Yet human only needs to eat a trace of selenium every day, about 55 micrograms or millionths of a gram (Lauren, 2012). Selenium is a constituent of seleno-proteins, many of which have important functions, including antioxidant protection, energy metabolism and redox regulation during transcription and gene expression (Kong et al., 2005). Selenium supplementation to plants enhances the production and quality of edible plant products, by increasing antioxidant activity of plants, as shown in tea leaves (Xu et al., 2003) and rice $(\mathrm{Xu}$ and $\mathrm{Hu}, 2004)$. In wheat plant, nutrient uptake, under salt stress, was better when adding selenium by soaking or soaking + foliar application, but in root the results were different depending on the concerned nutrient (Mona et al., 2011). This effect of Se could be attributed to its ability to reduce oxygen radicals produced in the presence of salinity stress by increasing the antioxidant enzymes activities that reduce the level of accumulation of reactive oxygen species (ROS) (Walaa et al, 2010). 
Humic acid is a bio-stimulant that is derived from leonardite shale and is among the most concentrated organic materials available. Elemental analysis of humic acid has shown it to consist largely of carbon and oxygen (about 50\% and $40 \%$, respectively). It also contains hydrogen (about 5\%), nitrogen (about 3\%), phosphorus and sulfur (both less than 1\%). Humic acid is a complex of closely related macromolecules. These molecules range in size from less than 1000 to more than 100,000 daltons, with the lower mass representing the younger material. Humic acid increases nutrient uptake, drought tolerance, and seed germination. It increases the microbial activity in the soil, making it an excellent root stimulator. Humic acid increases the availability of nutrients in applied fertilizers and those already initially existing in the soil. It helps to aerate the soil from the inside. It also helps to relatively lower the $\mathrm{pH}$ of the soil and flushes high levels of salts out of the root zone, all of which will help to promote better plant health and growth (Vrain, 2004). When applied to the clay soils, humic acid help breaking up compacted soils, allowing for enhanced water penetration as well as better root zone growth and development. When applied to the sandy soils, humic acid adds essential organic material necessary for water retention thus improving root growth and enhancing the sandy soil's ability to retain and not leach out vital plant nutrients (LLC, 2013).

Khaled et al. (2011) studied the effect of different levels of humic acids on the nutrient content and plant growth, along with soil properties under conditions of salinity. They found that soil application of humus increased the $\mathrm{N}$ uptake by corn plants; foliar application of humic acids increased the uptake of $\mathrm{P}, \mathrm{K}, \mathrm{Mg}$, $\mathrm{Na}, \mathrm{Cu}$ and $\mathrm{Zn}$. Although the effect of interaction between salt and soil humus application was found statistically significant, the interaction effect between salt and foliar humic acids treatment was not found significant. Under salt stress, the first doses of both soil and foliar application of humic substances increased the uptake of nutrients.

\section{Materials and Methods}

The present investigation was carried out to study the response of wheat plants to selenium application in presence of humic acid under salt stress conditions.

\section{Experimental}

The first experiment

The aim of this experiment was to study the effect of different concentrations from (sodium selenite- humic acid) on:

wheat grains germination, length of produced shoot and root along with their fresh and dry weights and soluble sugar percentage.

Wheat grains (Gemayzeh 9) unresisting to salinity were divided into (9) equal groups with (30 grains) in each group. The grains were soaked in the following treatments: distilled watersodium selenite $(5 \mu \mathrm{m} / 1-10 \mu \mathrm{m} / 1-20 \mu \mathrm{m} / 1-40 \mu \mathrm{m} /$ 1- $80 \mu \mathrm{m} / \mathrm{l})$ and humic acid $(0.5 \mathrm{gm} / 1000 \mathrm{mel}-1$ gm/1000mel- 2 gm/1000mel) for 6 hours. Grains germination was evaluated after one week, the length of shoots and roots as well as their fresh and dry weights and soluble sugar percentage.

\section{The second experiment}

This experiment was carried out to study the effect of the same previously mentioned concentrations from (sodium selenite) and concentrations from humic acid on soluble sugar percentage in wheat grains.

Wheat grains (Gemayzeh 9) unresisting to salinity were divided into 11 equal groups, (30 grains) in each group. Such grains were soaked in the following solutions: distilled water (as control)- sodium selenite $(5 \mu \mathrm{m} / 1-10 \mu \mathrm{m} / 1-20 \mu \mathrm{m} /$ 1- $40 \mu \mathrm{m} / 1-80 \mu \mathrm{m} / 1)$ and humic acid (1gm- $5 \mathrm{gm}-$ $10 \mathrm{gm}$ - $20 \mathrm{gm}-40 \mathrm{gm} / 100 \mathrm{mel}$ ) for $6 \mathrm{hr}$. After one week from germination, the soluble sugar percentage was measured.

\section{The third experiment}

This experiment was carried out to study the effect of different mixtures of sodium selenite with the best obtained suitable concentrations from humic acid, giving the highest soluble sugar percentage. Wheat grains (Gemayzeh 9) unresisting to salinity were divided into 10 equal groups, (30 grains) in each group. The previous wheat grains were soaked in the following :

- Distilled water (as control)

- $\operatorname{Se}(5 \mu \mathrm{m} / \mathrm{l})$.

- $\operatorname{Se}(10 \mu \mathrm{m} / \mathrm{l})$

- $\operatorname{Se}(20 \mu \mathrm{m} / \mathrm{l})$.

- Sodium selenite $(5 \mu \mathrm{m} / 1)+$ Humic acid (1 $\mathrm{gm} / 100 \mathrm{ml}$ )

- Sodium selenite $(5 \mu \mathrm{m} / \mathrm{l})+$ Humic acid (5 $\mathrm{gm} / 100 \mathrm{ml}$ ) 
- $\quad$ Sodium selenite $(10 \mu \mathrm{m} / \mathrm{l})+$ Humic acid (1 $\mathrm{gm} / 100 \mathrm{ml}$ )

- $\quad$ Sodium selenite $(10 \mu \mathrm{m} / \mathrm{l})+$ Humic acid (5 $\mathrm{gm} / 100 \mathrm{ml}$ )

- Sodium selenite $(20 \mu \mathrm{m} / \mathrm{l})+$ Humic acid (1 $\mathrm{gm} / 100 \mathrm{ml}$ )

- Sodium selenite $(20 \mu \mathrm{m} / \mathrm{l})+$ Humic acid (5 $\mathrm{gm} / 100 \mathrm{ml})$.

After one week from germination, the soluble sugar percentage was measured.

\section{The fourth experiment}

The aim of this experiment was to study the effect of the mixtures obtained from the last experiment on wheat grains germination under salt stress condition to be compared to the use of wheat grains (Sakha 94) resisting to salinity and also, wheat grains (Gemayzeh 9) unresisting to salinity were divided into 10 equal groups, (20 grains) in each group.

The previous wheat grains were soaked in the followings :

- Distilled water.

- Sodium selenite $(5 \mu \mathrm{m} / \mathrm{l})$.

- Sodium selenite $(5 \mu \mathrm{m} / \mathrm{l})+$ Humic acid (1 gm/100 ml).

- Sodium selenite $(5 \mu \mathrm{m} / \mathrm{l})+$ Humic acid (5 $\mathrm{gm} / 100 \mathrm{ml}$ ).

- $\quad$ Sodium selenite $(10 \mu \mathrm{m} / \mathrm{l})$.

- Sodium selenite $(10 \mu \mathrm{m} / \mathrm{l})+$ Humic acid (1 $\mathrm{gm} / 100 \mathrm{ml}$ )

- Sodium selenite $(10 \mu \mathrm{m} / \mathrm{l})+$ Humic acid (5 $\mathrm{gm} / 100 \mathrm{ml}$ )

- Sodium selenite $(20 \mu \mathrm{m} / \mathrm{l})$.

- $\quad$ Sodium selenite $(20 \mu \mathrm{m} / \mathrm{l})+$ Humic acid (1 $\mathrm{gm} / 100 \mathrm{ml}$ )

- Sodium selenite $(20 \mu \mathrm{m} / \mathrm{l})+$ Humic acid (5 $\mathrm{gm} / 100 \mathrm{ml}$ )

Samples of 20 soaked wheat grains, were planted in $200 \mathrm{~g}$ pure sand pots for 2 weeks. After two weeks, the plants were irrigated from the nutrient solution $(\mathrm{EC}=2 \mathrm{dS} / \mathrm{m})$ then the plants were irrigated from either the nutrient solution (E.C $=5 \mathrm{dS} / \mathrm{m})$ or nutrient solution $(\mathrm{EC}=10 \mathrm{dS} / \mathrm{m})$. Mingled nutrient solution adjust its salinity (5 - $10 \mathrm{dS} / \mathrm{m}$ ) using $\mathrm{NaCl}$.

After 4 weeks of the start of this experiments, the shoots were cut to then evaluate fresh and dry weights as well as length of both shoot and root, $\% \mathrm{Na}, \mathrm{K}$ and proline.

\section{The fifth experiment}

The aim of this experiment was to apply the best concentrations obtained from the fourth experiment to the field. Wheat grains were cultivated in saline soil through 10 plots with an area of $\left(140 \mathrm{~m}^{2}\right)$; the treatments were distributed in these plots as follows :

- The first plot was cultivated with wheat grains (Gemayzeh 9) unresisting to salinity, the grains were soaked in distilled water and planting in non-saline plot.

- The second plot was cultivated with wheat grains (Gemayzeh 9) of a saline soil, the grains being previously soaked in distilled water.

- The third plot was cultivated with wheat grains (Sakha 94) resisting to salinity. , the grains being previously soaked in distilled water.

- The fourth plot was cultivated with wheat grains (Suds 1) resisting to salinity., the grains being previously soaked in distilled water.

The rest of the plots were subjected to experimental treatments following grains were previously soaked for six hours in either :

- Sodium selenite $(5 \mu \mathrm{m} / \mathrm{l})$.

- Sodium selenite $(5 \mu \mathrm{m} / \mathrm{l})+$ Humic acid (1 $\mathrm{gm} / 100 \mathrm{ml}$ ).

- Sodium selenite $(10 \mu \mathrm{m} / \mathrm{l})$.

- Sodium selenite $(10 \mu \mathrm{m} / \mathrm{l})+$ Humic acid (1 $\mathrm{gm} / 100 \mathrm{ml}$ )

- Sodium selenite $(20 \mu \mathrm{m} / \mathrm{l})$.

- Sodium selenite $(20 \mu \mathrm{m} / \mathrm{l})+$ Humic acid (1 $\mathrm{gm} / 100 \mathrm{ml}$ )

Samples from developed plants were collected at (15 -30 -45 -60 -75 -90) day from cultivation.

At each stage of wheat growth the followings were evaluated :

- Fresh and dry weight of plants.

- Nitrogen, Sodium, Potassium and selenium percentage in plants.

- The proportion of nitrogen and protein in the crop output.

- The amount of the crop output attributed to the acre.

Methods of analysis

Soil analysis

Soil samples were collected before plant cultivation, air dried, ground and sieved through 
a $2 \mathrm{~mm}$ sieve, finally preserved for the following analysis according to Jackson (1973) and Baruah \& Barthakur (1997).

Soil mechanical analysis was carried out by the pipette method using sodium hexametaphosphate as a dispersing agent.

$\mathrm{pH}$ was measured in the soil suspension (1:2.5 ratio) using a combined electrode connected to a $\mathrm{pH}$ meter.

EC was measured in the soil extract (1: 2.5 ratio) using electrical conductivity bridge.

Total carbonates were determined volumetrically using Collin's calcimeter and calculated as calcium carbonate.

TABLE 1. Some physical and chemical analyses of the studied soil sample.

\begin{tabular}{|c|c|}
\hline \multicolumn{2}{|l|}{ Particle size distribution, $\%$} \\
\hline C. Sand & 4.60 \\
\hline F. Sand & 12.5 \\
\hline Silt & 37.3 \\
\hline Clay & 45.6 \\
\hline Texture class & Clay \\
\hline $\mathrm{CaCO}_{3}, \%$ & 6.50 \\
\hline $\mathrm{pH}(1: 2.5$ soil:water suspension) & 8.13 \\
\hline $\mathrm{EC}_{\mathrm{e}}, \mathrm{dS} \mathrm{m} \mathrm{m}^{-1}$ & 5.60 \\
\hline \multicolumn{2}{|l|}{ Soluble cations, meq $\mathrm{L}^{-1}$} \\
\hline $\mathrm{Ca}^{2+}$ & 17.1 \\
\hline $\mathrm{Mg}^{2+}$ & 12.8 \\
\hline $\mathrm{Na}^{+}$ & 22.1 \\
\hline $\mathrm{K}^{+}$ & 3.10 \\
\hline \multicolumn{2}{|l|}{ Soluble anions, meq $\mathbf{L}^{-1}$} \\
\hline $\mathrm{CO}_{3}^{2-}$ & 0.00 \\
\hline $\mathrm{HCO}_{3}^{-}$ & 5.30 \\
\hline $\mathrm{Cl}^{-}$ & 30.1 \\
\hline $\mathrm{SO}_{4}^{2-}$ & 19.6 \\
\hline \multicolumn{2}{|l|}{ Concerned elements, $\mathrm{mg} \mathrm{kg}^{-1}$} \\
\hline $\mathrm{N}$ & 141 \\
\hline $\mathrm{P}$ & 8.34 \\
\hline K & 302 \\
\hline $\mathrm{Se}$ & 0.00 \\
\hline
\end{tabular}

Egypt. J. Soil Sci. 57, No. 2 (2017)
Soluble calcium and magnesium were determined by titration with Na2EDTA.

Soluble sodium and potassium were determined using Flame photometer.

Soluble carbonate and bicarbonate were determined by titration with $\mathrm{HCl}$.

Soluble chloride was determined by titration with silver nitrate.

Soluble sulphates were calculated by subtracting the total soluble anions $\left(\mathrm{Cl}^{-}, \mathrm{CO}_{3}{ }^{-2}\right.$ and $\mathrm{HCO}_{3}^{-}$) from total soluble cations.

Plant analysis:

The plant samples dried at $70^{\circ} \mathrm{C}$ were wet digested with a mixture of $\mathrm{H}_{2} \mathrm{SO}_{4}$ and $\mathrm{H}_{2} \mathrm{O}_{2}$ according to Cottenie et al. (1982).

Total nitrogen content in plant was determined by micro kjeldah method using $5 \%$ boric acid and $40 \% \mathrm{NaOH}$ as described by Black et al. (1965).

Total sodium and potassium were determined using Flame photometer (Jackson ,1973).

Total selenium was determined by atomic absorption according to Chapman and Pratt (1961).

The protein in wheat plant was calculated by multiplying $\% \mathrm{~N}$ by 5.75 according to A.O.A.C. (1980)

Total proline was extracted according to A.O.A.C. (1980).

\section{Statistical analysis}

Obtained data were subjected to analyses of variance (ANOVA) and the differences among the means were obtained using the Denken test using M-Stat computer program according to Snedecor and Cocharn (1980).

Results

First experiment

Results showed that Nither selenium or humic acid significantly affected, compared to the control, the germination of the studied grains (Table 2). On the other hand, a significant increase was obtained for both shoots and roots length compared to the control, when using Se and humic acid concentrations.Fresh and dry 
TABLE 2. Effect of selenium and humic acid on germination, total soluble sugar and growth of wheat plants

\begin{tabular}{|lcccccc|}
\hline Treatments & $\begin{array}{c}\text { no. of } \\
\text { grains }\end{array}$ & $\begin{array}{c}\text { length } \\
\text { of shoot } \\
\text { cm }\end{array}$ & $\begin{array}{c}\text { length of } \\
\text { root cm }\end{array}$ & $\begin{array}{c}\text { Fresh } \\
\text { weight g }\end{array}$ & $\begin{array}{c}\text { Dry weight } \\
\text { g }\end{array}$ & $\begin{array}{c}\text { Total soluble } \\
\text { sugar mg/f.w }\end{array}$ \\
\hline Control & $47.67 \mathrm{abc}$ & $3.17 \mathrm{cbd}$ & $4.2 \mathrm{bc}$ & $5.9 \mathrm{ba}$ & $2 \mathrm{a}$ & $12.9 \mathrm{bdc}$ \\
\hline Se $5 \mu \mathrm{m}$ & $45 \mathrm{c}$ & $3.2 \mathrm{cb}$ & $3.9 \mathrm{bdc}$ & $5.8 \mathrm{ba}$ & $1.9 \mathrm{bac}$ & $17.1 \mathrm{bac}$ \\
\hline Se $10 \mu \mathrm{m}$ & $48.3 \mathrm{ab}$ & $3.9 \mathrm{~b}$ & $4.9 \mathrm{abc}$ & $6.6 \mathrm{a}$ & $1.9 \mathrm{bac}$ & $26.9 \mathrm{a}$ \\
\hline Se $20 \mu \mathrm{m}$ & $47 \mathrm{abc}$ & $3.4 \mathrm{cb}$ & $4.4 \mathrm{abc}$ & $5.2 \mathrm{bc}$ & $1.9 \mathrm{bac}$ & $27.1 \mathrm{a}$ \\
\hline Se $40 \mu \mathrm{m}$ & $47.7 \mathrm{abc}$ & $3.4 \mathrm{cb}$ & $4.2 \mathrm{bc}$ & $5.1 \mathrm{bc}$ & $1.9 \mathrm{bac}$ & $22 \mathrm{a}$ \\
\hline Se $80 \mu \mathrm{m}$ & $46 \mathrm{bc}$ & $3.6 \mathrm{cb}$ & $4.3 \mathrm{bc}$ & $5.2 \mathrm{bc}$ & $1.9 \mathrm{bac}$ & $20.3 \mathrm{ab}$ \\
\hline humic $0.5 \mathrm{~g} / 1000 \mathrm{mel}$ & $50 \mathrm{a}$ & $4.9 \mathrm{a}$ & $5.3 \mathrm{ba}$ & $7 \mathrm{a}$ & $1.9 \mathrm{bac}$ & $11.5 \mathrm{bdc}$ \\
\hline humic $1 \mathrm{~g} / 1000 \mathrm{mel}$ & $49.3 \mathrm{a}$ & $5 \mathrm{a}$ & $5.8 \mathrm{a}$ & $5.1 \mathrm{bc}$ & $1.5 \mathrm{bdc}$ & $26.2 \mathrm{a}$ \\
\hline humic $2 \mathrm{~g} / 1000 \mathrm{mel}$ & $49.3 \mathrm{a}$ & $2.7 \mathrm{~cd}$ & $2.7 \mathrm{ed}$ & $4.5 \mathrm{~cd}$ & $1.9 \mathrm{bac}$ & $17.9 \mathrm{bac}$ \\
\hline
\end{tabular}

Means with the same letter are not significantly different.

weight of developed plants followed a trend almost similar to that of germination. Either Se or humic acid was favorable but not significantly affecting, compared to the control. Finally, all Se concentrations as well as those of humic acid were mostly favorable, total soluble sugars significantly being obtained from Se 10, 20 and $40 \mu \mathrm{m}$ and humic acid $1 \mathrm{~g} / \mathrm{l}$ (Table 2).

TABLE 3. Effect of selenium and humic acid on total soluble sugar

\begin{tabular}{|c|c|}
\hline $\operatorname{Se} 5 \mu \mathrm{m}$ & $377.89 \mathrm{a}$ \\
\hline $\mathrm{Se} 10 \mu \mathrm{m}$ & $374.43 \mathrm{a}$ \\
\hline $\mathrm{Se} 20 \mu \mathrm{m}$ & $301.83 \mathrm{a}$ \\
\hline $\mathrm{Se} 40 \mu \mathrm{m}$ & $267.50 \mathrm{a}$ \\
\hline $\mathrm{Se} 80 \mu \mathrm{m}$ & $261.36 \mathrm{a}$ \\
\hline humic $1 \mathrm{~g} / 100 \mathrm{mel}$ & $121.98 \mathrm{~b}$ \\
\hline humic $5 \mathrm{~g} / 100 \mathrm{mel}$ & $113.32 \mathrm{~b}$ \\
\hline humic $10 \mathrm{~g} / 100 \mathrm{mel}$ & $105.45 \mathrm{~b}$ \\
\hline humic $20 \mathrm{~g} / 100 \mathrm{mel}$ & $93.63 \mathrm{~b} \mathrm{c}$ \\
\hline humic $40 \mathrm{~g} / 100 \mathrm{mel}$ & $82.93 \mathrm{~b} \mathrm{c}$ \\
\hline
\end{tabular}

\section{Second experiment}

Content of total soluble sugar increased when using selenium concentrations of $(5 \mu \mathrm{m}-10 \mu \mathrm{m})$ compared to these of $(20 \mu \mathrm{m}-40 \mu \mathrm{m}-80 \mu \mathrm{m})$, higher values being obtained by humic acid concentrations (1gm -5gm) /100 mel ) compared to other concentrations (Table 3 ).

\section{Third experiment}

Results revealed positive favorable response for the total soluble sugar content by application of Se + humic acid, Se $5+1 \mathrm{~g}$ humic acid being superior (Table 4).

TABLE 4. Effect of selenium with humic acid on Total soluble sugar

\begin{tabular}{|lc|}
\hline & \\
\hline Control & $34.2 \mathrm{bc}$ \\
\hline Se5 $\mu \mathrm{m}$ & $47.8 \mathrm{ab}$ \\
\hline Se10 $\mu \mathrm{m}$ & $46.9 \mathrm{ab}$ \\
\hline Se20 $\mu \mathrm{m}$ & $43.3 \mathrm{ab}$ \\
\hline Se5+humic $1 \mathrm{~g}$ & $53.6 \mathrm{a}$ \\
\hline Se5+humic $5 \mathrm{~g}$ & $52.8 \mathrm{a}$ \\
\hline Se10+humic $1 \mathrm{~g}$ & $42.7 \mathrm{ab}$ \\
\hline Se10+humic $5 \mathrm{~g}$ & $50.7 \mathrm{a}$ \\
\hline Se20+humic $1 \mathrm{~g}$ & $52.1 \mathrm{a}$ \\
\hline Se20+humic $5 \mathrm{~g}$ & $52.3 \mathrm{a}$ \\
\hline
\end{tabular}

\section{Fourth experiment}

For the first level of salinity (A) there was no significant difference between the fresh weight of control sensitive and that resistant. Application of both Se and humic acid was favorable for both fresh and dry weights of the studied plants. For the second level of salinity (B) there was no 
significant difference between the fresh weight of control sensitive and that resistant one. Results showed superiority of Se $5+\mathrm{h} 1$ for salinity resistance (Tables 5\&6).

With regard to length of shoot and root under the first level of salinity conditions there was no significant difference between the shoot length of the control. For the second level of salinity there was no significant difference between both fresh and dry weights of control sensitive plants. But it was noted that there was a significant increase in the fresh weight of all other solutions $(\mathrm{se} 5+\mathrm{h} 1$ - se10+h1 - se20+h1 - se20+h5) except (control

TABLE 5. Effect of selenium with humic acid on fresh and dry weights-shoot and root length as well as proline and porn $\mathrm{K}$ and $\mathrm{Na}$ contents under the first level of salinity (A)

\begin{tabular}{|l|c|c|c|c|c|c|c|}
\hline \multicolumn{1}{|c|}{ Treatments } & $\begin{array}{c}\text { Fresh } \\
\text { Weight }\end{array}$ & $\begin{array}{c}\text { Dry } \\
\text { weight }\end{array}$ & $\begin{array}{c}\text { Shoot } \\
\text { length }\end{array}$ & $\begin{array}{c}\text { Root } \\
\text { length }\end{array}$ & Proline & K\% & Na\% \\
\hline con & $1.7 \mathrm{bc}$ & $0.6 \mathrm{c}$ & $16.5 \mathrm{~b}$ & $8.5 \mathrm{a}$ & $8.4 \mathrm{~d}$ & $0.4 \mathrm{a}$ & $0.17 \mathrm{c}$ \\
\hline con+sa.A & $4.3 \mathrm{a}$ & $1.8 \mathrm{a}$ & $19.5 \mathrm{a}$ & $9 \mathrm{a}$ & $34.9 \mathrm{a}$ & $0.4 \mathrm{a}$ & $0.81 \mathrm{a}$ \\
\hline conR & $1.9 \mathrm{bc}$ & $0.3 \mathrm{c}$ & $17.5 \mathrm{a}$ & $6 \mathrm{ab}$ & $18.8 \mathrm{ab}$ & $0.29 \mathrm{ab}$ & $0.2 \mathrm{~b}$ \\
\hline conR+ sa.A & $3.9 \mathrm{a}$ & $2 \mathrm{a}$ & $18.5 \mathrm{a}$ & $5 \mathrm{ab}$ & $21.5 \mathrm{~b}$ & $0.4 \mathrm{a}$ & $0.9 \mathrm{a}$ \\
\hline se5+ sa.A & $4.1 \mathrm{a}$ & $1.5 \mathrm{ab}$ & $19 \mathrm{a}$ & $7 \mathrm{a}$ & $31.8 \mathrm{a}$ & $0.4 \mathrm{a}$ & $0.9 \mathrm{a}$ \\
\hline se5+h1+ sa.A & $5.5 \mathrm{a}$ & $0.6 \mathrm{c}$ & $18.5 \mathrm{a}$ & $8.5 \mathrm{a}$ & $21.5 \mathrm{~b}$ & $0.5 \mathrm{a}$ & $0.92 \mathrm{a}$ \\
\hline se5+h5+ sa.A & $2.7 \mathrm{~b}$ & $1.4 \mathrm{ab}$ & $18 \mathrm{a}$ & $5.5 \mathrm{~b}$ & $14.2 \mathrm{c}$ & $0.5 \mathrm{a}$ & $0.9 \mathrm{a}$ \\
\hline se10+ sa.A & $3.5 \mathrm{ab}$ & $1.5 \mathrm{ab}$ & $16.5 \mathrm{~b}$ & $6 \mathrm{ab}$ & $11.1 \mathrm{c}$ & $0.5 \mathrm{a}$ & $0.97 \mathrm{a}$ \\
\hline se10+h1+ sa.A & $4.1 \mathrm{a}$ & $1.2 \mathrm{~b}$ & $18 \mathrm{a}$ & $5.5 \mathrm{~b}$ & $11.7 \mathrm{c}$ & $0.22 \mathrm{~b}$ & $0.9 \mathrm{a}$ \\
\hline se10+h5+ sa.A & $3.2 \mathrm{ab}$ & $1.2 \mathrm{~b}$ & $20.5 \mathrm{a}$ & $6 \mathrm{ab}$ & $18.9 \mathrm{~b}$ & $0.3 \mathrm{ab}$ & $1.03 \mathrm{a}$ \\
\hline se20+ sa.A & $3.3 \mathrm{ab}$ & $1.4 \mathrm{ab}$ & $18 \mathrm{a}$ & $7 \mathrm{a}$ & $19.2 \mathrm{~b}$ & $0.26 \mathrm{~b}$ & $0.6 \mathrm{~b}$ \\
\hline se20+h1+ sa.A & $4.2 \mathrm{a}$ & $1.9 \mathrm{a}$ & $20.5 \mathrm{a}$ & $8.5 \mathrm{a}$ & $21.9 \mathrm{~b}$ & $0.3 \mathrm{ab}$ & $0.9 \mathrm{a}$ \\
\hline se20+h5+ sa.A & $2.8 \mathrm{~b}$ & $1.04 \mathrm{bc}$ & $21 \mathrm{a}$ & $5.5 \mathrm{~b}$ & $19.2 \mathrm{~b}$ & $0.5 \mathrm{a}$ & $0.8 \mathrm{a}$ \\
\hline
\end{tabular}

TABLE 6. Effect of selenium with humic acid on fresh and dry weights-shoot and root length along with \% proline, $\mathrm{K}$ and $\mathrm{Na}$ of developed plants under the second level of salinity (B)

\begin{tabular}{|l|c|c|c|c|c|c|c|}
\hline \multicolumn{1}{|c|}{ Treatments T } & $\begin{array}{c}\text { Fresh } \\
\text { Weight }\end{array}$ & $\begin{array}{c}\text { Dry } \\
\text { weight }\end{array}$ & $\begin{array}{c}\text { Shoot } \\
\text { leanth }\end{array}$ & $\begin{array}{c}\text { Root } \\
\text { lenth }\end{array}$ & Proline & K\% & Na\% \\
\hline con & $1.7 \mathrm{~b}$ & $0.6 \mathrm{~b}$ & $16.5 \mathrm{~b}$ & $8.5 \mathrm{a}$ & $8.4 \mathrm{jk}$ & $0.4 \mathrm{bc}$ & $0.17 \mathrm{hi}$ \\
\hline con+sa.B & $2.4 \mathrm{~b}$ & $0.8 \mathrm{bc}$ & $16 \mathrm{~b}$ & $7 \mathrm{a}$ & $50.9 \mathrm{ab}$ & $0.17 \mathrm{hi}$ & $1.2 \mathrm{bc}$ \\
\hline conR & $1.9 \mathrm{~b}$ & $0.3 \mathrm{c}$ & $17.5 \mathrm{a}$ & $6 \mathrm{a}$ & $18.8 \mathrm{ef}$ & $0.29 \mathrm{~cd}$ & $0.2 \mathrm{gh}$ \\
\hline conR+ sa.B & $3.2 \mathrm{a}$ & $1.2 \mathrm{~b}$ & 14.5 & $8.5 \mathrm{a}$ & $24.6 \mathrm{~cd}$ & $0.26 \mathrm{de}$ & $1.01 \mathrm{bc}$ \\
\hline se5+ sa.B & $2.1 \mathrm{~b}$ & $0.6 \mathrm{bc}$ & $15 \mathrm{~b}$ & $6.5 \mathrm{a}$ & $25.1 \mathrm{~cd}$ & $0.26 \mathrm{de}$ & $1.1 \mathrm{bc}$ \\
\hline se5+h1+ sa.B & $5.7 \mathrm{a}$ & $3.1 \mathrm{a}$ & $18 \mathrm{a}$ & $6.5 \mathrm{a}$ & $30.1 \mathrm{bc}$ & $0.29 \mathrm{~cd}$ & $1.2 \mathrm{bc}$ \\
\hline se5+h5+ sa.B & $3.8 \mathrm{ab}$ & $0.9 \mathrm{~b}$ & $13 \mathrm{c}$ & $6.5 \mathrm{a}$ & $36.5 \mathrm{ab}$ & $0.5 \mathrm{bc}$ & $0.83 \mathrm{~cd}$ \\
\hline se10+ sa.B & $2.9 \mathrm{ab}$ & $0.8 \mathrm{~b}$ & $14.5 \mathrm{c}$ & $7 \mathrm{a}$ & $28.9 \mathrm{bc}$ & $0.22 \mathrm{fh}$ & $1.1 \mathrm{bc}$ \\
\hline se10+h1+ sa.B & $3.4 \mathrm{ab}$ & $1.6 \mathrm{a}$ & $17.5 \mathrm{a}$ & $7 \mathrm{a}$ & $20.6 \mathrm{de}$ & $0.24 \mathrm{ef}$ & $1.2 \mathrm{bc}$ \\
\hline se10+h5+ sa.B & $4.5 \mathrm{a}$ & $1.2 \mathrm{~b}$ & $16.5 \mathrm{~b}$ & $8.5 \mathrm{a}$ & $30.2 \mathrm{bc}$ & $0.15 \mathrm{ij}$ & $1.2 \mathrm{bc}$ \\
\hline se20+ sa.B & $3.3 \mathrm{ab}$ & $0.9 \mathrm{~b}$ & $14 \mathrm{c}$ & $6.5 \mathrm{a}$ & $26.6 \mathrm{bc}$ & $0.11 \mathrm{jk}$ & $0.57 \mathrm{fg}$ \\
\hline se20+h1+ sa.B & $5.1 \mathrm{a}$ & $2.9 \mathrm{a}$ & $19 \mathrm{a}$ & $8.5 \mathrm{a}$ & $26.2 \mathrm{bc}$ & $0.3 \mathrm{~cd}$ & $0.9 \mathrm{bc}$ \\
\hline se20+h5+ sa.B & $4 \mathrm{a}$ & $1.9 \mathrm{a}$ & $19 \mathrm{a}$ & $5.5 \mathrm{~b}$ & $35.5 \mathrm{ab}$ & $0.19 \mathrm{hi}$ & $0.84 \mathrm{~cd}$ \\
\hline
\end{tabular}


resistant-se $5+$ h5 - se $10-$ se20) where a significant decrease compared to the control sensor, It was also noted that there was no significant difference in dry weight for both sensitive and resistant control with both of the previous solutions with the exception of $(\mathrm{se} 20+\mathrm{h} 5)$ where a significant decrease compared to the control event.

Potassium content : For the first level of salinity $(5 \mathrm{dS} / \mathrm{m})$ there was no significant difference between the potassium content of control sensitive and resistant and between both of the solutions used except a mixture of $(\mathrm{Se} 10+\mathrm{h} 1$ - se20) where the reduction in the potassium content has happened is significant compared to the control.

For the second level of salinity there was no significant difference between the potassium content of control sensitive with (se10 - se10+h5 - se20+h5), But it was noted that there was a significant increase in the potassium content of all other solutions (control resistant - se 5 - se $5+\mathrm{h} 1-$ se $5+$ h5 -se10+h1 - se20+h1) except (se20) where a significant decrease compared to the control sensor.

Sodium content : For the first and second levels of salinity there was no significant difference between the sodium content of control sensitive and resistant and between both of the solutions used except a mixture of (se20) where the reduction in the sodium content has happened is significant compared to the control.

Proline : For the first level of salinity there was no significant difference between the proline of control sensitive plants and (se5), but it was noted that there was a significant decrease in the proline of all other solutions and resistant control (Tables 7\&8).

For the second level of salinity there was no significant difference between the proline of control sensitive and the treatments $(\mathrm{se} 5+\mathrm{h} 1$ - se $5+\mathrm{h} 5$ - se $10-\operatorname{se} 10+\mathrm{h} 5-\mathrm{se} 20-\mathrm{se} 20+\mathrm{h} 1$

TABLE 7. Effect of selenium with humic acid on fresh weight of the studied plants

\begin{tabular}{|l|c|c|c|c|c|c|}
\hline \multicolumn{1}{|c|}{ Treatments } & $\mathbf{1 5}$ day & $\mathbf{3 0}$ day & $\mathbf{4 5}$ day & $\mathbf{6 0}$ day & $\mathbf{7 5}$ day & $\mathbf{9 0}$ day \\
\hline con & $1.655 \mathrm{a}$ & $2.075 \mathrm{a}$ & $2.25 \mathrm{a}$ & $3.35 \mathrm{a}$ & $4.7 \mathrm{ab}$ & $10.84 \mathrm{a}$ \\
\hline con-soak & $1.85 \mathrm{a}$ & $1.625 \mathrm{a}$ & $2 \mathrm{a}$ & $3.2 \mathrm{a}$ & $5.45 \mathrm{ab}$ & $9.875 \mathrm{a}$ \\
\hline conR1 & $1.675 \mathrm{a}$ & $1.145 \mathrm{a}$ & $2.3 \mathrm{a}$ & $4.11 \mathrm{a}$ & $5.7 \mathrm{ab}$ & $8.03 \mathrm{a}$ \\
\hline conR2 & $2.025 \mathrm{a}$ & $2.225 \mathrm{a}$ & $2.4 \mathrm{a}$ & $3.885 \mathrm{a}$ & $8.7 \mathrm{a}$ & $9.945 \mathrm{a}$ \\
\hline se5 & $1.155 \mathrm{a}$ & $1.655 \mathrm{a}$ & $2.7 \mathrm{a}$ & $2.765 \mathrm{ab}$ & $7.35 \mathrm{a}$ & $8.95 \mathrm{a}$ \\
\hline se5+h1 & $0.815 \mathrm{ab}$ & $2.455 \mathrm{a}$ & $2.6 \mathrm{a}$ & $3.765 \mathrm{a}$ & $8.75 \mathrm{a}$ & $11.5 \mathrm{a}$ \\
\hline se10 & $1.64 \mathrm{a}$ & $2.585 \mathrm{a}$ & $2.15 \mathrm{a}$ & $2.83 \mathrm{ab}$ & $3.9 \mathrm{~b}$ & $8.795 \mathrm{a}$ \\
\hline se10+h1 & $0.985 \mathrm{a}$ & 1.08 & $2.1 \mathrm{a}$ & $1.34 \mathrm{~b}$ & $3.65 \mathrm{~b}$ & $7.87 \mathrm{ab}$ \\
\hline se20 & $1.81 \mathrm{a}$ & $1.22 \mathrm{a}$ & $1.1 \mathrm{a}$ & $2.36 \mathrm{ab}$ & $5.4 \mathrm{ab}$ & $6.43 \mathrm{ab}$ \\
\hline se20+h1 & $0.765 \mathrm{ab}$ & $1.715 \mathrm{a}$ & $2.35 \mathrm{a}$ & $3.465 \mathrm{a}$ & $4.25 \mathrm{ab}$ & $9.22 \mathrm{a}$ \\
\hline
\end{tabular}

TABLE 8. Effect of selenium with humic acid on dry weight

\begin{tabular}{|l|c|c|c|c|c|c|}
\hline Treatments & $\mathbf{1 5}$ day & $\mathbf{3 0}$ day & $\mathbf{4 5}$ day & $\mathbf{6 0}$ day & $\mathbf{7 5}$ day & $\mathbf{9 0}$ day \\
\hline con & $0.375 \mathrm{a}$ & $0.22 \mathrm{a}$ & $0.395 \mathrm{a}$ & $0.51 \mathrm{a}$ & $0.92 \mathrm{a}$ & $2.2 \mathrm{a}$ \\
\hline con-soak & $0.315 \mathrm{a}$ & $0.26 \mathrm{a}$ & $0.36 \mathrm{a}$ & $0.565 \mathrm{a}$ & $1.17 \mathrm{a}$ & $2.075 \mathrm{a}$ \\
\hline conR1 & $0.325 \mathrm{a}$ & $0.17 \mathrm{ab}$ & $0.355 \mathrm{a}$ & $0.78 \mathrm{a}$ & $1.09 \mathrm{a}$ & $1.975 \mathrm{a}$ \\
\hline conR2 & $0.34 \mathrm{a}$ & $0.345 \mathrm{a}$ & $0.335 \mathrm{a}$ & $0.64 \mathrm{a}$ & $1.835 \mathrm{a}$ & $2.24 \mathrm{aa}$ \\
\hline se5 & $0.225 \mathrm{a}$ & $0.27 \mathrm{a}$ & 0.4 & $0.595 \mathrm{a}$ & $1.49 \mathrm{a}$ & $2.16 \mathrm{a}$ \\
\hline se5+h1 & $0.155 \mathrm{ab}$ & $0.375 \mathrm{a}$ & $0.41 \mathrm{a}$ & $0.61 \mathrm{a}$ & $2.35 \mathrm{a}$ & $1.635 \mathrm{a}$ \\
\hline se10 & $0.345 \mathrm{a}$ & $0.39 \mathrm{a}$ & $0.495 \mathrm{a}$ & $0.6 \mathrm{a}$ & $0.79 \mathrm{a}$ & $1.74 \mathrm{a}$ \\
\hline se10+h1 & $0.265 \mathrm{a}$ & $0.2 \mathrm{a}$ & $0.43 \mathrm{a}$ & $0.275 \mathrm{ab}$ & $0.875 \mathrm{a}$ & $1.915 \mathrm{a}$ \\
\hline se20 & $0.325 \mathrm{a}$ & $0.19 \mathrm{ab}$ & $0.26 \mathrm{ab}$ & $0.565 \mathrm{a}$ & $1.31 \mathrm{a}$ & $1.6 \mathrm{a}$ \\
\hline se20+h1 & $0.145 \mathrm{ab}$ & $0.305 \mathrm{a}$ & $0.4 \mathrm{a}$ & $0.67 \mathrm{a}$ & $0.995 \mathrm{a}$ & $2.475 \mathrm{a}$ \\
\hline
\end{tabular}

Egypt. J. Soil Sci. 57, No.2 (2017) 
TABLE 9. Effect of selenium with humic acid on nitrogen content

\begin{tabular}{|l|c|c|c|c|c|c|}
\hline Treatments & $\mathbf{1 5}$ & $\mathbf{3 0}$ & $\mathbf{4 5}$ & $\mathbf{6 0}$ & $\mathbf{7 5}$ & $\mathbf{9 0}$ \\
\hline con & $2.597 \mathrm{a}$ & $1.5687 \mathrm{ab}$ & $3.255 \mathrm{ab}$ & $3.325 \mathrm{a}$ & $1.82 \mathrm{ab}$ & $1.435 \mathrm{ab}$ \\
\hline con-soak & $2.604 \mathrm{a}$ & $2.2295 \mathrm{a}$ & $2.1 \mathrm{~b}$ & $3.955 \mathrm{a}$ & $1.995 \mathrm{ab}$ & $1.715 \mathrm{a}$ \\
\hline conR1 & $2.247 \mathrm{a}$ & $2.8693 \mathrm{a}$ & $2.681 \mathrm{ab}$ & $3.99 \mathrm{a}$ & $2.261 \mathrm{a}$ & $1.645 \mathrm{a}$ \\
\hline conR2 & $2.765 \mathrm{a}$ & $1.925 \mathrm{a}$ & $4.725 \mathrm{a}$ & $2.52 \mathrm{ab}$ & $2.044 \mathrm{a}$ & $1.085 \mathrm{ab}$ \\
\hline se5 & $2.254 \mathrm{a}$ & $2.3569 \mathrm{a}$ & $4.235 \mathrm{a}$ & $2.905 \mathrm{a}$ & $2.345 \mathrm{a}$ & $1.386 \mathrm{ab}$ \\
\hline se5+h1 & $1.596 \mathrm{ab}$ & $2.7587 \mathrm{a}$ & $5.075 \mathrm{a}$ & $2.744 \mathrm{ab}$ & $1.211 \mathrm{ab}$ & $1.89 \mathrm{a}$ \\
\hline se10 & $2.527 \mathrm{a}$ & $3.1836 \mathrm{a}$ & $2.555 \mathrm{ab}$ & $2.975 \mathrm{a}$ & $2.24 \mathrm{a}$ & $1.82 \mathrm{a}$ \\
\hline se10+h1 & $1.694 \mathrm{ab}$ & $1.6268 \mathrm{ab}$ & $3.15 \mathrm{ab}$ & $2.87 \mathrm{a}$ & $1.365 \mathrm{ab}$ & $0.805 \mathrm{~b}$ \\
\hline se20 & $2.667 \mathrm{a}$ & $1.7962 \mathrm{ab}$ & $1.295 \mathrm{~b}$ & $1.225 \mathrm{ab}$ & $1.386 \mathrm{ab}$ & $1.26 \mathrm{ab}$ \\
\hline se20+h1 & $1.414 \mathrm{ab}$ & $2.6201 \mathrm{a}$ & $3.36 \mathrm{ab}$ & $3.22 \mathrm{a}$ & $1.673 \mathrm{ab}$ & $1.19 \mathrm{ab}$ \\
\hline
\end{tabular}

TABLE 10. Effect of selenium with humic acid on potasium content

\begin{tabular}{|l|c|c|c|c|c|c|}
\hline Treatments & $\mathbf{1 5}$ & $\mathbf{3 0}$ & $\mathbf{4 5}$ & $\mathbf{6 0}$ & $\mathbf{7 5}$ & $\mathbf{9 0}$ \\
\hline con & $1.3168 \mathrm{a}$ & $1.8389 \mathrm{a}$ & $2.5056 \mathrm{bc}$ & $2.0688 \mathrm{ab}$ & $0.9516 \mathrm{a}$ & $1.564 \mathrm{~b}$ \\
\hline con-soak & $1.48 \mathrm{a}$ & $1.3468 \mathrm{ab}$ & $1.8832 \mathrm{c}$ & $1.4736 \mathrm{~b}$ & $1.014 \mathrm{a}$ & $1.816 \mathrm{~b}$ \\
\hline conR1 & $1.096 \mathrm{a}$ & $1.02675 \mathrm{ab}$ & $1.88 \mathrm{c}$ & $5.3 \mathrm{a}$ & $1.074 \mathrm{a}$ & $1.2716 \mathrm{~b}$ \\
\hline conR2 & $1.0384 \mathrm{a}$ & $1.8389 \mathrm{a}$ & $3.3536 \mathrm{bc}$ & $3.016 \mathrm{ab}$ & $1.194 \mathrm{a}$ & $1.3708 \mathrm{~b}$ \\
\hline se5 & $1.024 \mathrm{a}$ & $1.3912 \mathrm{ab}$ & $3.6032 \mathrm{~b}$ & $1.432 \mathrm{~b}$ & $0.828 \mathrm{a}$ & $1.24016 \mathrm{~b}$ \\
\hline se5+h1 & $0.9024 \mathrm{a}$ & $2.1423 \mathrm{a}$ & $7.7575 \mathrm{a}$ & $3.7088 \mathrm{ab}$ & $1.0728 \mathrm{a}$ & $1.672 \mathrm{~b}$ \\
\hline se10 & $1.328 \mathrm{a}$ & $2.886 \mathrm{a}$ & $2.12425 \mathrm{bc}$ & $3.0368 \mathrm{ab}$ & $1.1328 \mathrm{a}$ & $2.152 \mathrm{~b}$ \\
\hline se10+h1 & $1.0944 \mathrm{a}$ & $0.9808 \mathrm{ab}$ & $2.2504 \mathrm{bc}$ & $3.328 \mathrm{ab}$ & $1.188 \mathrm{a}$ & $0.696 \mathrm{c}$ \\
\hline se20 & $1.328 \mathrm{a}$ & $1.3536 \mathrm{ab}$ & $1.3775 \mathrm{c}$ & $1.1508 \mathrm{~b}$ & $0.876 \mathrm{a}$ & $6.568 \mathrm{a}$ \\
\hline se20+h1 & $0.9952 \mathrm{a}$ & $1.496 \mathrm{ab}$ & $2.13875 \mathrm{bc}$ & $1.5728 \mathrm{~b}$ & $1.0584 \mathrm{a}$ & $1.236 \mathrm{~b}$ \\
\hline
\end{tabular}

TABLE 11. Effect of selenium with humic acid on sodium content

\begin{tabular}{|l|c|c|c|c|c|c|}
\hline Treatments & $\mathbf{1 5}$ & $\mathbf{3 0}$ & $\mathbf{4 5}$ & $\mathbf{6 0}$ & $\mathbf{7 5}$ & $\mathbf{9 0}$ \\
\hline con & $0.5058 \mathrm{a}$ & $0.2988 \mathrm{ab}$ & $0.3195 \mathrm{ab}$ & $0.3213 \mathrm{a}$ & $0.22655 \mathrm{ab}$ & $0.2635 \mathrm{ab}$ \\
\hline con-soak & $0.6138 \mathrm{a}$ & $0.3294 \mathrm{a}$ & $0.40257 \mathrm{ab}$ & $0.3876 \mathrm{a}$ & $0.23 \mathrm{ab}$ & $0.3723 \mathrm{a}$ \\
\hline conR1 & $0.4716 \mathrm{a}$ & $0.2088 \mathrm{ab}$ & $0.35145 \mathrm{ab}$ & $0.3638 \mathrm{a}$ & $0.305 \mathrm{a}$ & $0.3876 \mathrm{a}$ \\
\hline conR2 & $0.4572 \mathrm{a}$ & $0.4608 \mathrm{a}$ & $0.64539 \mathrm{a}$ & $0.3689 \mathrm{a}$ & $0.4785 \mathrm{a}$ & $0.3774 \mathrm{a}$ \\
\hline se5 & $0.1818 \mathrm{~b}$ & $0.306 \mathrm{a}$ & $0.54741 \mathrm{a}$ & $0.2941 \mathrm{ab}$ & $0.3277 \mathrm{a}$ & $0.3349 \mathrm{a}$ \\
\hline se5+h1 & $0.2934 \mathrm{~b}$ & $0.3492 \mathrm{a}$ & $0.727395 \mathrm{a}$ & $0.2652 \mathrm{ab}$ & $0.2842 \mathrm{ab}$ & $0.3434 \mathrm{a}$ \\
\hline se10 & $0.4698 \mathrm{a}$ & $0.3978 \mathrm{a}$ & $0.510135 \mathrm{a}$ & $0.3791 \mathrm{a}$ & $0.47125 \mathrm{a}$ & $0.3621 \mathrm{a}$ \\
\hline se10+h1 & $0.3366 \mathrm{ab}$ & $0.2556 \mathrm{ab}$ & $0.62835 \mathrm{a}$ & $0.4981 \mathrm{a}$ & $0.6003 \mathrm{a}$ & $0.1666 \mathrm{~b}$ \\
\hline se20 & $0.5076 \mathrm{a}$ & $0.261 \mathrm{ab}$ & $0.29181 \mathrm{~b}$ & $0.3009 \mathrm{a}$ & $0.38135 \mathrm{a}$ & $0.3587 \mathrm{a}$ \\
\hline se20+h1 & $0.3096 \mathrm{ab}$ & $0.3438 \mathrm{a}$ & $0.473925 \mathrm{ab}$ & $0.408 \mathrm{a}$ & $0.25955 \mathrm{ab}$ & $0.3145 \mathrm{a}$ \\
\hline
\end{tabular}

Egypt. J. Soil Sci. 57, No. 2 (2017) 
- se20+h5), but it was noted that there was a significant decrease in the proline of (resistant control - se5 - se10+h1).

\section{Field experiments}

Dry and fresh weight: In general there has been no noticeable significant difference in both dry and fresh weights between control and other studied treatments (Tables 7, 8, 9, 10, 11).

Nitrogen and potassium content: The highest percentage of nitrogen and potassium contents in the studied plant was recorded at the age of 45 days with a general no significance except when using (se5 - se5+h1).

Sodium content : The highest percentage of sodium content in the studied plant was recorded at the age of 15 days, results being generally not significant except when using (se $5-\mathrm{se} 5+\mathrm{h} 1)$.

\section{Crop output}

Grain weight per ardap/acre:

Using the (se5 - se $5+\mathrm{h} 1)$ treatment was favorable; an increase in grain weight is significantly obtained compared to the control (Table 12).

TABLE 12. Effect of selenium with humic acid on grains

\begin{tabular}{|l|c|c|c|c|c|c|c|}
\hline \multicolumn{1}{|c|}{ Treatments } & $\begin{array}{c}\text { Balsnabul } \\
\text { straw weight }\end{array}$ & $\begin{array}{c}\text { weight of } \\
\text { grains }\end{array}$ & $\begin{array}{c}\text { weight of } \\
\text { grains/acre }\end{array}$ & $\begin{array}{c}\text { Yield/ } \\
\text { alardb }\end{array}$ & N\% & $\begin{array}{c}\text { Protine } \\
\text { \% }\end{array}$ & $\begin{array}{c}\text { Se } \boldsymbol{\mu g} / \\
\text { Kg }\end{array}$ \\
\hline con & $14.75 \mathrm{a}$ & $5.25 \mathrm{a}$ & $3150 \mathrm{a}$ & $21 \mathrm{a}$ & $1.6 \mathrm{ab}$ & $9.6 \mathrm{ab}$ & $102 \mathrm{de}$ \\
\hline con-soak & $10.75 \mathrm{bc}$ & $3.4 \mathrm{de}$ & $2010 \mathrm{de}$ & $13.4 \mathrm{ed}$ & $1.39 \mathrm{ab}$ & $8 \mathrm{ab}$ & $366 \mathrm{~d}$ \\
\hline conR1 & $8.25 \mathrm{~cd}$ & $3.4 \mathrm{de}$ & $2025 \mathrm{de}$ & $13.5 \mathrm{ed}$ & $1.5 \mathrm{ab}$ & $8.8 \mathrm{ab}$ & $266 \mathrm{~d}$ \\
\hline conR2 & $10 \mathrm{bc}$ & $4.13 \mathrm{bc}$ & $2475 \mathrm{bc}$ & $16.5 \mathrm{bc}$ & $1.27 \mathrm{~b}$ & $7.3 \mathrm{~b}$ & $575 \mathrm{c}$ \\
\hline se5 & $12 \mathrm{ab}$ & $5.13 \mathrm{a}$ & $3075 \mathrm{a}$ & $20.5 \mathrm{a}$ & $1.8 \mathrm{ab}$ & $10.3 \mathrm{ab}$ & $989 \mathrm{ab}$ \\
\hline se5+h1 & $10 \mathrm{bc}$ & $4.4 \mathrm{ab}$ & $2625 \mathrm{ab}$ & $17.5 \mathrm{ab}$ & $2.12 \mathrm{a}$ & $12.2 \mathrm{a}$ & $1640 \mathrm{a}$ \\
\hline se10 & $13 \mathrm{ab}$ & $5 \mathrm{a}$ & $3000 \mathrm{a}$ & $20 \mathrm{ab}$ & $1.38 \mathrm{~b}$ & $7.9 \mathrm{~b}$ & $1403 \mathrm{a}$ \\
\hline se10+h1 & $6.75 \mathrm{fg}$ & $3.4 \mathrm{de}$ & $2025 \mathrm{de}$ & $13.5 \mathrm{ed}$ & $1.7 \mathrm{ab}$ & $9.9 \mathrm{ab}$ & $958 \mathrm{ab}$ \\
\hline se20 & $6.75 \mathrm{fg}$ & $3 \mathrm{e}$ & $1800 \mathrm{e}$ & $12 \mathrm{e}$ & $1.8 \mathrm{ab}$ & $10.3 \mathrm{ab}$ & $1817 \mathrm{a}$ \\
\hline se20+h1 & $7.5 \mathrm{df}$ & $3.3 \mathrm{de}$ & $1950 \mathrm{de}$ & $13 \mathrm{ed}$ & $1.6 \mathrm{ab}$ & $9.1 \mathrm{ab}$ & $1130 \mathrm{a}$ \\
\hline
\end{tabular}

\section{Protein percentage}

In spite of no significant difference among treatments, the protein content in the studied plants was relatively better when using (se5 se $5+$ h1 - se10 - se20 - se20+h1) treatments compared to the control.

\section{Selenium content in seeds}

Generally, increasing rate applied from selenium increased significantly its content in grains compared to the control.

\section{Discussion}

Response of germination and growth to Se and Humic acid under natural conditions:

Generally, only low concentrations of applied selenium led to improve growth. These responses are similar to those obtained by Azadeh Saffaryazdi et al. (2012) with spinach (Spinacia oleracea L.) plants (Hartikainen et al., 2000) with ryegrass
(Yang and Ding, 2000) with tobacco and (Xue et al., 2000) with lettuce as well as with potato (Turakainen et al., 2004). At lower concentrations, selenium stimulated growth, on the other hand, at high doses act as pro-oxidant, reducing yields and inducing metabolic disturbances (Azadeh Saffaryazdi et al., 2012). High Se levels may inhibit photosynthesis, impair nutrient uptake and transport (Kahle, 1988). Hawrylak (2008) showed that disturbances of growth and reduction of plant's biomass at the presence of high selenium concentrations in the nutrient solution may have resulted from the disturbance of mineral balance of plants, namely accumulation of large amounts of phosphorus in shoot tissues of maize.

Selenium at the lowest level $(1 \mathrm{mg} / \mathrm{L})$ stimulated spinach growth parameters like shoot and root fresh weight, shoot and root dry weight, total dry weight of plants as well as shoot and root length; higher Se levels, on the other hand, dramatically reduced those growth parameters. Low Se level 
may be required by this accumulator plant as a micronutrient and may increase its antioxidative capacity. Moreover, selenium enhanced nutrients and Se uptake in spinach leaves which is more useful for human health (Azadeh, 2012).

It was also noted that the use of humic acid led to improve the growth possibly due mainly to the humic acid increasing grain germination, nutrient uptake and drought tolerance. It increases the microbial activity in the soil, making it an excellent root stimulator. Humic acid increases the availability of nutrients in fertilizers and in those already existing initially in soil. It helps to aerate the soil. It also helps to lower the $\mathrm{pH}$ of the soil and flushes salts out of the root zone, all of which will help to promote better plant health and growth. When Humic Acids enter plants at early stages of development, they increase cell division and root development thus hastening establishment. Also, humic acid acts as a natural chelator enhancing the availability of iron thus increased photosynthesis and sugar production and increased storage for plant defense(LLC, 2013).

\section{Germination and growth under saline conditions}

Se has been shown to counteract various abiotic stresses induced in plants by cold, drought, high light intensity, water stress, salinity and heavy metals but the associated mechanisms are rather complicated and still remain to be fully elucidated (Renwei et al., 2013).

Selenium is a constituent of seleno-proteins, many of which have important functions, including antioxidant protection, energy metabolism and redox regulation during transcription and gene expression Kong et al. (2005). Selenium supplementation to plants enhance the production and quality of edible plant products, by increasing antioxidant activity of plants, as shown in tea leaves (Xu et al., 2003 and rice (Xu et al., 2004).

In fact, results showed that the use of (se 5 - se $5+\mathrm{h} 1)$ led to an increase in the absorption of $(\mathrm{N}-\mathrm{K})$ and low $\mathrm{Na}$ absorption under salt stress conditions. Yassen et al. (2011) reported that Se interaction with plants depended on its concentration. At lower rates, Se stimulated growth of ryegrass seedlings; at high doses, however, it acted as pro-oxidant reducing yields and inducing metabolic disturbances the effect of irrigation with saline water on $\mathrm{N}, \mathrm{P}$ and $\mathrm{K}$ contents in leaves of eggplants under Se supplements, compared with the control (without any treatments) during the two studied seasons. Increasing salinity resulted in increased $\mathrm{N}$ and $\mathrm{P}$ contents in the leaves, but decreased K. Almost, $\mathrm{N}, \mathrm{P}$ and $\mathrm{K}$ contents in leaves increased with increasing Se supplements up to $20 \mu \mathrm{M}$ then decreased with higher concentrations. Regarding the interaction between irrigation water salinity and Se supplements, Se $20 \mu \mathrm{M}$ with all saline water treatments generally gave the highest value of $\mathrm{N}, \mathrm{P}$ and $\mathrm{K}$ contents in plant leaves (AbuElsoud and Abd-Elrahman, 2016).

Increasing $\mathrm{N}$ and $\mathrm{P}$ contents in leaves and fruits of eggplant with increasing salinity of irrigation water may be due to increase the amino acids inside the plant with increasing the stress; amino acids also interact with phospholipids to adjust the osmotic potential according to Walaa et al. (2010). Se had a high ability to induce antioxidant and hormone balance in plants.

Decreasing K content with increasing salinity of irrigation water, on the other hand, may be due to the increase of $\mathrm{NaCl}$ concentration; $\mathrm{Na}+$ content increased in leaves and fruits indicating that the eggplant (which has a glycophytic reaction) could not control uptake of $\mathrm{Na}+$ (Akinci et al., 2004).

The proline content in plant fresh leaves increased with increasing salinity of irrigation water (indication of stress), but decreased with increasing Se supplements compared to the control, these findings agreed with those obtained by Walaa et al. (2010).

Humic acids used as a supplement can be effective in enhancing antioxidation enzymatic activities; appearance of effects is retarded because of decomposition and release of auxinlike compounds from HAs by organic acids from the plant roots (Kenya et al., 2015).

Hamide et al. (2015) showed that humic acid and salicylic acid significantly improvement factors under salinity. The results revealed that concentrations of SA and HU had significant effects on germination percentage and radical.

Humic acid added to saline soil significantly improved the variables affected by high salinity and also increased plant nitrate, nitrogen and phosphorus, reduced soil electrical conductivity, 
proline and electrolyte leakage of plant, enhanced plant root and shoot dry weight by allowing nutrients and water to be released to the plant as needed. Humic acid has great potential in alleviating salinity stress on plant growth and growth parameters in saline soils of arid and semi-arid areas. This humic acid appeared to be highly effective for soil conditioners in vegetable growth, to improve crop tolerance and growth under saline conditions (Adil et al., 2012).

Ounia et al. (2014) showed a beneficial effect of humic acid in salt affected rhizosphere likely due to a 'direct' action on plant together with an 'indirect action' on the metabolism of soil microorganisms, the dynamics of uptake of soil nutrients and soil physical conditions.

It was observed from the above results that when using selenium with humic acid most notably (se5+humic 1g/100mel) led to improved germination rate and total production as well as improve the quality of the crop output by increasing the protein content. It was also noted from previous results that when soak before planting wheat in concentrations of selenium (se5,se10and se20) led to an increase in the proportion of selenium in crop output compared to the control under salt stress conditions, Perhaps the reason is soaking wheat grain in selenium before planting leads to increased grain content of selenium, as a result of element movement from the high concentration (external solution) to the low concentration (a grain of wheat), also was humic to add to the impact of selenium significantly increase selenium in crop output compared to the control.

The application of low concentrations of $\mathrm{Se}$ with moderate amounts of humic acid caused the highest antioxidant activity, the same effect of application of high Se concentrations with no humic acid (Ghasemia, 2015).

Hasanuzzaman (2011) suggested that the exogenous application of Se rendered the plants more tolerant to salt stress-induced oxidative damage by enhancing their antioxidant defense and methylglyoxal (MG) detoxification systems. Manal et al. (2012) studied the exogenous application of $\mathrm{Se}$ or $\mathrm{Si}$ and reported that they enhanced the drought tolerance of rice seedlings. The mechanism of defense was mediated by increasing some osmolytes particularly in roots of stressed seedlings.

\section{Referances}

Adil ,A., Kant, C. andTuran, M. (2012) Humic acid application alleviates salinity stress of bean (Phaseolus vulgaris L.) plants decreasing membrane leakage. African Journal of Agricultural Research, 7 (7), pp. 1073-1086, 19 February.

Akinci, I.E., Akinci, S., Yilmaz, K. and Dikici, H. (2004) Response of eggplant varieties (Solanum melongena) to salinity in germination and seedling stages. New Zealand J. Crop Hort. Sci. 2004; 32, 193-200.

A.O.A.C.(1980) Official Methods of Analysis (13ed) Association of Official Analytical Chemists Washington.

Baruah, T.C and Barthakur, H.P. (1997) "A Text Book of Soil Analysis". Vikas Publishing Housing, PVTLTD, New Delhi.

Black, C. A. (1965) "Methods of Soil Analysis. Part 2. Chemical and Microbiological Properties " $2^{\text {nd }}$ ed. Am. Sco. Agron. Madison, Wisconsin, USA

Chapman,H.D. andPratt, P.E. (1961) Methods of Analysis for Soil, Plant and Waters. Univ. Calif. Division of Agric. Sci.,pp.16-38,97-99 and 161174.

Cottenie, A.; Verloo, M.; . Kiekens, L; Velgh, G. and Camerlynech, R. (1982) "Chemical Analysis of Plants and Soils." State Univ Ghent, Belgium.

Kahle, H. (1988) The effects of lead and cadmium on the growth and mineral balance of young beech Fagus sylvatica L. in sand culture. Dissertationes Botanicae 127, Berlin: J. Cra- mer.

Hamide,F. ,Ameri, A., Mohammadi, S. and Astaraee, A. (2015) Influence of salicylic acid \& humic acid on salinity stress tolerance during seed germination of (lens culinaris medik. journal of Current Research in Science (issn 2322-5009) coden (USA): jcrsdj 2013, Vol. 1, No. 5, pp.396-399.

Hartikainen H., Xue, T. and Piironen, V. (2000) Selenium as an anti-oxidant and pro-oxidant in ryegrass. Plant and Soil, 225, 193-200.

Hawrylak, N. B. (2008) Effect of selenium on selected ma- cronutrients in maize plants. J. Elementol. 13 (4), 513-519.

Egypt. J. Soil Sci. 57, No.2 (2017) 
Jackson, M. L. (1973) "Soil Chemical Analysis". Prentice-Hall of india Private Limited, New Delhi.

Kenya, N., Wanga, B., Nishiyaa, K., Ushijimaa, K., Zhua, Q., Masami Fukushimaa and Toshiharu Ichijob (2015) Effects of humic acids derived from lignite and cattle manure on antioxidant enzymatic activities of barley root. Journal of Environmental Science and Health, Part B: Pesticides, Food Contaminants, and Agricultural Wastes

Khaled, H. and Fawy, H. A. (2011) Effect of different levels of humic acids on the nutrient content, plant growth, and soil properties under conditions of salinity. Soil and Water Research, Vol. 6 No. 1 pp. 21-29.

Ghasemia, K., Bolandnazara, S., Tabatabaeia, S.J., Pirdashtib, H., Arzanlouc,M., Ebrahimzadehd,M.A and Fathid. H. (2015) Antioxidant properties of garlic as affected by selenium and humic acid treatments. New Zealand Journal of Crop and Horticultural Science, Volume 43, Issue 3, 2015.

Kong L, Wang M. and Bi, D. (2005) Selenium modulates the activities of antioxidant enzymes, osmotic homeostasis and promotes the growth of sorrel seedlings under salt stress. Plant Growth Regulation, 45, 155-163.

Lauren Cox. (2012) Health Benefits of Selenium. Centre for Research in Ecotoxicology and Environmental Remediation, Institute of Agro-Environmental Protection, the Ministry of Agriculture.

LLC. (2013) Copyright Natural Environmental Systems.

M. A. Abul-Soud and Shaimaa H. Abd-Elrahman (2015) Foliar Selenium Application to Improve the Tolerance of Eggplant Grown under Salt Stress Conditions. DOI: 10.9734/IJPSS/2015/19992.

Manal M. E., Khattab, H. I. and Helal, N. M. (2012) Effects of silicon or selenium on photosynthetic apparatus and antioxidant capacity of rice plant grown under drought condition. Egypt. J. Exp. Biol. (Bot.). 2012; 8(2), 271-283

Mirza H., Mohammad A. H. and Fujita, M.I. (2011) Selenium-nduced Up-Regulation of the Antioxidant Defense and Methylglyoxal Detoxification System Reduces Salinity-Induced Damage in Rapeseed
Seedlings.Biological Trace Element Research. December 2011, Volume 143, Issue 3, pp 17041721.

M.M. TAHIR, M. KHURSHID, M.Z. KHAN, M.K. ABBASI and M.H. KAZMI (2011) LigniteDerived Humic Acid Effect on Growth of Wheat Plants in Different Soils.

Mona, I., Nossier, Sh.M., Gawish, H.E. Abu-Hussin, M. and Mubark, M. (2011) Effects of selenium on some plant nutrient contents under salt stress conditions. J. Bio.Chem.Environ. Sci., 6 (2), 355366.

Renwei Fenga, Chaoyang Weic and Shuxin Tu (2013) The roles of selenium in protecting plants against abiotic stresses. Environmental and Experimental Botany, Volume 87, March 2013, Pages 58-68.

Senedeccor G.W. andW.G.Cocharn, W.G. (1980) Statistical Methods. $7^{\text {th }}$ ed., Iowa stat Univ. Press. Amr.USA,pp.255-269.

Turakainen M., Hartikainen, H. and Seppanen, M. M. (2004) Effects of selenium treatments on potato (Solanum tuberosum L.) growth and concentrations of soluble sugars and starch. J. Agric. Food Chem. 52 (17), 5378-5382.

Vrain Arbor Care (2004) info@stvrainarborcare.com • flash player

Walaa, A. El-Shalakany, Shatlah, M. A., Atteia, M.H. and Sror,H.A.M. (2010) Selenium induces antioxidant defensive enzymes and promotes tolerance aganist alinity stress in cucumber seedlings (Cucumis sativus), Arab Univ. J. Agric. Sci., Ain Shams Univ., Cairo, 18 (1), 65-76.

Xu, J., Yang, F., Chen L., Hu, Y. and Hu, Q. (2003) Effect of selenium on increasing the antioxidant activity of tea leaves harvested during the early spring tea producing season. J. Agric. Food Chem. 2003; 51, 1081-1084.

Xu, J. and Hu, Q. (2004) Effect of foliar application of selenium on the antioxidant activity of aqueous and ethanolic extracts of selenium-enriched rice. $J$. Agric. Food Chem. 2004; 52, 1759-1763.

Yassen A. A., Safia M. Adam and Sahar M. Zaghloul (2011) Impact of nitrogen fertilizer and foliar spray of selenium on growth, yield and chemical 
of potato plants. Aust. J. Basic \& Appl. Sci. 2011;5:1296-1303 .

Ounia, Y., Ghnayaa, T., Montemurrob, F., Abdellya, Ch. and Lakhdara, A. (2014) The role of humic substances in mitigating the harmful effects of soil salinity and improve plant productivity. International Journal of Plant Production, 8 (3), July 2014 ISSN: 1735-6814.

Yang, L.F. and Ding, R.X. (2000) Effects of selenium application on selenium content and distribution in flue-cured tobacco grown on Se-low soils. Journal of Nanjing Agricultural University, 23, 47-50.

(Received: 3/2/2016; accepted:30/3/2016)

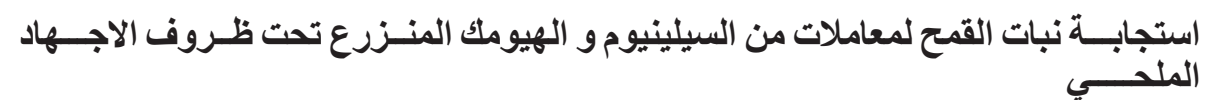

منى ابراهيم نصير ، شريف محمود جاويش ، طايع عبد اللطيف طه و منال مبارك

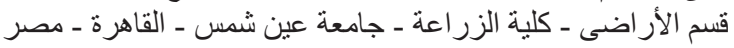

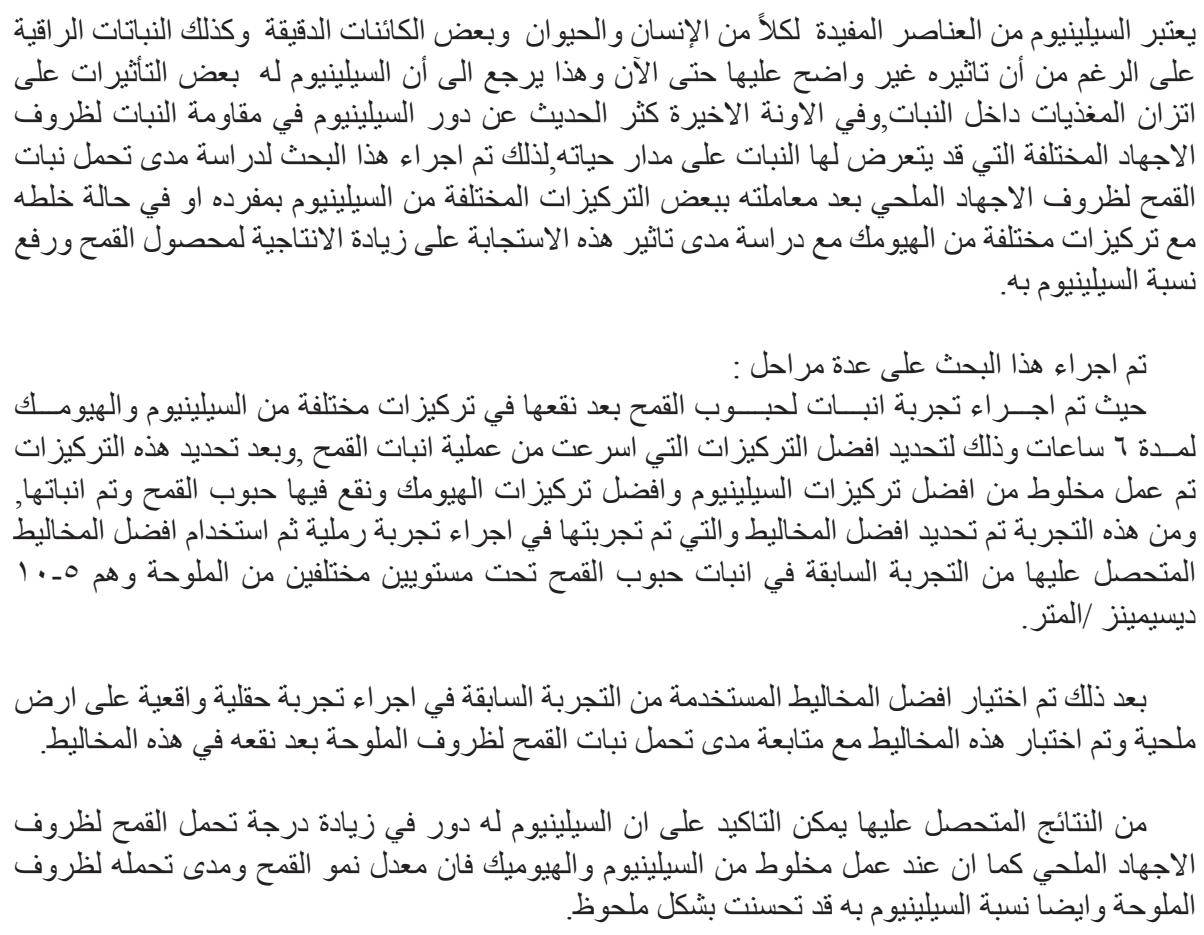

